\title{
Different Approaches for the Head Fire Perimeter Definition in Wildland Fires
}

\author{
EULALIA PLANAS, MIQUEL CUBELLS, and ELSA PASTOR \\ Center for Technological Risk Studies (CERTEC) \\ Universitat Politècnica de Catalunya \\ ETSEIB. P-G, Planta 2. Av. Diagonal, 647. 08028 - Barcelona, Catalunya, Spain
}

\begin{abstract}
The perimeter of a wildland fire can always be split into different sections (i.e. head, flanks and back) according to their particular behaviour. The way to delimit the different sections of a fire perimeter depends firstly on the purpose and type of the analysis that is going to be carried out and secondly on the type of information available for that, but in summary we have observed that there is no bias-free and precise definition on the literature to delimit the head and the flanks of a fire perimeter which could be confidently used for scientific purposes. The aim of this paper was to explore a new definition to delimit the head fire perimeter that shall be objective, unequivocal, and adequate for scientific studies. Diverse options have been explored which have came out with five different criteria that can be used when analysing fire behaviour for scientific purposes. The use of one or other criteria will depend on the final aim of the study being undertaken.
\end{abstract}

KEYWORDS: wildfires, fire behaviour, IR.

\section{INTRODUCTION}

Wildland fire growth depends on several environmental factors such us weather, terrain and fuel conditions, thus giving different fire shape patterns and sizes. Nevertheless, a fire perimeter can always be split into different sections (i.e. head, flanks and back) according to their particular behaviour. The head of the fire is the section of the fire perimeter spreading at fastest velocities and highest intensities because it has a major alignment with the propagating forces whereas the flanks spread at lower velocities due the loss of alignment with any of the major factors (e.g. the wind). The rate of spread and intensity of the backfire is the lowest, since the rear part of the fire perimeter spreads against the main propagating factors, i.e. down slope and/or against the wind.

The split of the fire perimeter in its different sections (head, flanks and back) in terms of fire behaviour is useful and necessary for suppression tactics and communication (e.g. in terms of allocating aerial and ground resources, Fig. 1). Concerning technical and scientific purposes, this fragmentation is also required (e.g. in modelling and predicting fire behaviour at fine scales) because the behaviour of the fire can be completely different in terms of rate of spread and intensity within the fire perimeter. The characterization and understanding of this variability is essential to implement safer and more realistic fire management practices and to better predict the effect of fire on ecosystem components.

Several authors have proposed more accurate definitions when studying the fire growth. Among them we highlight the mathematical approaches based on a simple elliptical fire growth model [1] or on a double ellipse model [2]. These authors provided simple geometrical rules to delimit the head, flanks and back of the fire perimeter, which was supposed to assume a roughly regular shape. Although some of these approaches have been used to build wildfire simulation systems [3] they neither envisage the different physical phenomena that take place along the fire perimeter nor the complexity of a wildfire growth. Cheney and Gould [4] give two possible definitions to delimit the head of the fire following a more physical approach. They related the head of the fire to a) the length of the fire perimeter where the flames are leaning towards unburnt fuel, and also to b) the length of the fire perimeter that influences the next period of head fire spread, which has its limits where the isochrones (i.e. the lines that delimit the fire edge at certain time instants) start losing parallelism (which indicated flank spread) beginning to diverge rapidly (showing now head fire spread). These definitions were based on the observation of the fire behaviour from a scientific point of view; however they are not free of some degree of subjectivity. 


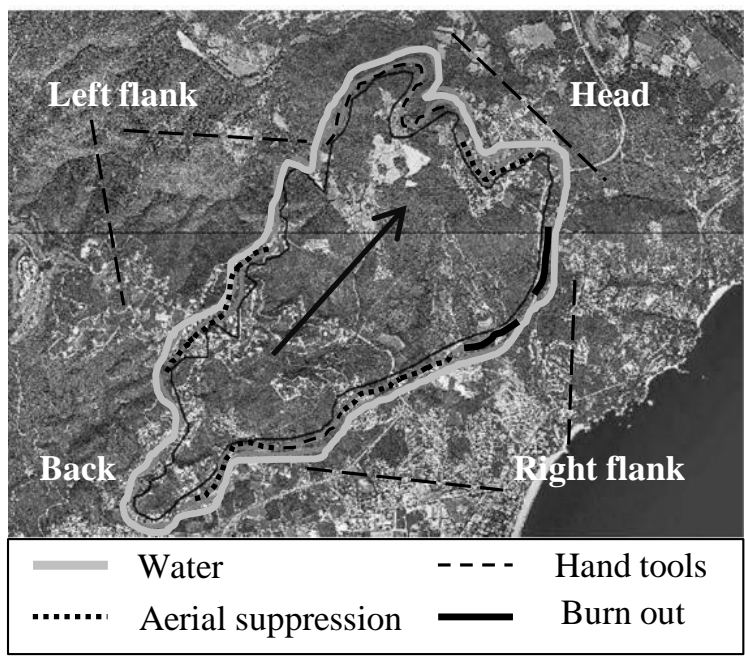

Fig. 1. Perimeter and area burnt (434.4 ha) at the Castell-Platja d'Aro wildland fire (Catalonia, Spain, 29/08/2003). The arrow indicates the direction of the head fire propagation. Source: Generalitat de

Catalunya.

The use of one or other approaches to delimit the different sections of a fire perimeter depends firstly on the purpose and type of the analysis that is going to be carried out and secondly on the type of information available for that, but in summary we have observed that there is no bias-free and precise definition in the literature to delimit the head and the flanks of a fire perimeter which could be confidently used for scientific purposes.

The aim of this paper is to explore new definitions to delimit the head fire perimeter that shall be objective, unequivocal, and adequate for scientific studies. The milestone of this work is to study the fire behaviour variability at very fine scale in shrubland fires within the framework of the experimental project FuSE which is devoted to study the fuel and fire dynamics of mallee ecosystems in South Australia [5].

\section{MATERIALS AND METHODS}

\section{Experimental Site and Design}

The experimental program by which this work has been developed was conducted at a site west of the private in-holding (Kirra Station) within the Ngarkat Conservation Park ( $\left.35^{\circ} 45 \mathrm{~S}, 140^{\circ} 51 \mathrm{E}\right)$, eastern South Australia on 3, 4, and 5 March 2008. This site was also being used for a project evaluating the effectiveness of aerial suppression tactics on wildfires using different chemical additives [6]. Ngarkat Conservation Park has a Mediterranean climate with warm, dry summers and cool wetter winters and experiences high intensity fires almost annually. Three large plots were prepared specifically for the aerial suppression experiments. They were made up of $20 \mathrm{yr}$ old woodland dominated by multi-stemmed eucalypts (mallee). Mallee woodlands are made up of short (2 to $10 \mathrm{~m}$ ) trees with shrubby understory. The surface fuel and suspended bark fuel are the main fuel layers that carry the flame front. These three plots were labelled AS1, AS2 and AS3, and were 52, 49, and 93 ha respectively. More detailed information concerning the experimental site and design of the experiments can be found in Ref. [5]. The experiment undertaken in Plot AS2 is the one specifically used to develop this work (Fig. 2). It was filmed from a helicopter with a standard video camera (visual spectrum) and an infrared camera. The cameras were positioned on top of a fixed tripod in the helicopter and were hand operated allowing focussing control. The infrared imagery was captured using an AGEMA Thermovision 570-Pro (FSI-FLIR Systems). This camera operated within the 7.5-13 $\mu \mathrm{m}$ range and was equipped with a frame grabber to control and store sequences of IR images $(240 \times 320$ pixels) onto a laptop computer at a rate of 5 frames per second. Each IR image is a $240 \times 320$ cell temperature matrix which is represented by a colour map gradient. The helicopter was positioned so that the majority of the plot was in view for the duration of each fire, allowing for fire behaviour and drops to be recorded and monitored. Due to the purpose of this paper only the first period of the experiment, before the start of the aerial suppression, has been used. 


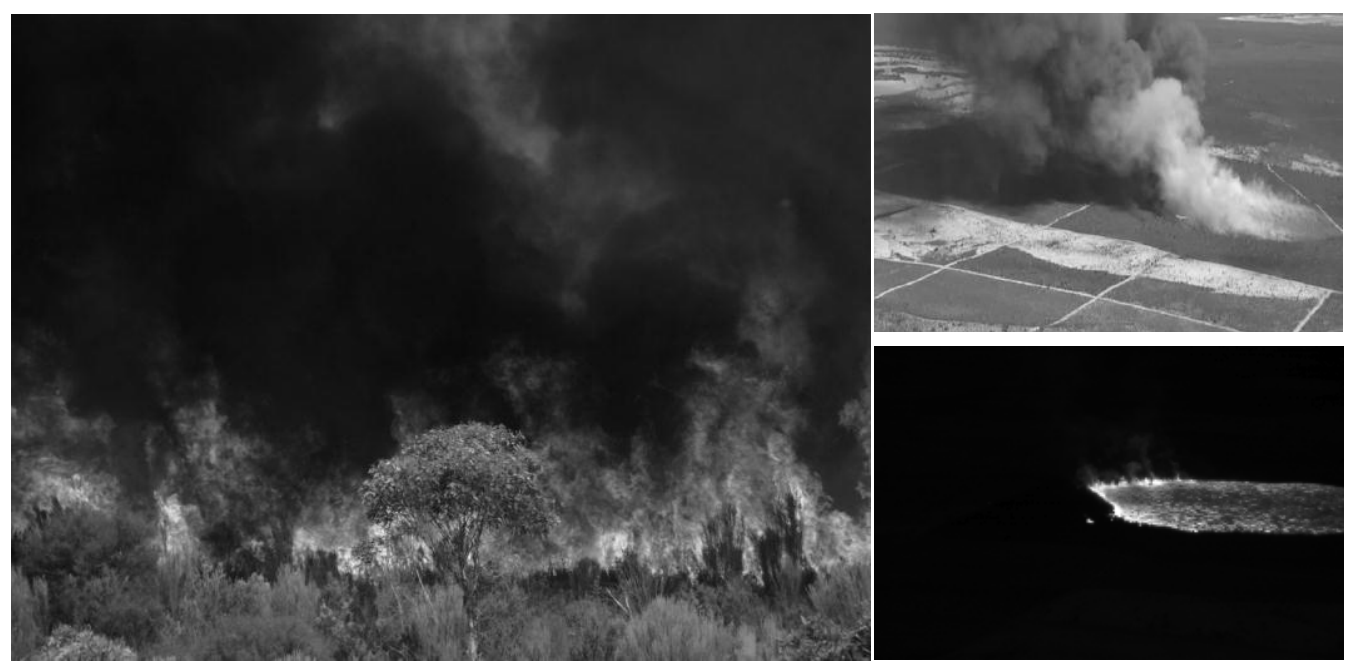

Fig. 2. Image of the fire front spreading in AS2 (left); video frame (right, top); IR frame of the fire perimeter growing in AS2 (right, bottom).

\section{Methodology for IR Analysis}

The images taken by the IR camera were used to obtain the position of the fire perimeter at a maximum frequency of one isochrone every $10 \mathrm{~s}$ allowing to draw an isochrones map and also to obtain the rate of spread (ROS) for each pixel of every isochrone. The methodology developed to quantitatively analyze these field experiments consists of a package of applications (algorithms and user-friendly interfaces) implemented using the MATLAB ${ }^{\circledR}$ computing software, and aiming to address different aspects of the aerial attack and the related fire behavior. The main characteristics of these applications have also been described elsewhere [7].

\section{RESULTS AND DISCUSSION}

From the head fire perimeter definitions already existing in the literature, the ones based on the theoretical elliptical shape of the fire are not adequate to analyze real fires as in reality, wildfires would hardly adopt this regular shape. Concerning the definitions given by Cheney and Gould [4], definition a) could not be used in this work due to the lack of images with enough resolution to detect the tilting direction of the flames. Taking into account the information available from the experiments, mainly made up of the isochrones maps and ROS, and the definition b) of the head fire perimeter given by Cheney and Gould, three main approaches have been undertaken by considering different criteria for the head fire perimeter selection:

1. Criteria established by using the maximum ROS value at every isochrone.

2. Criteria established by analyzing the derivative of the isochrones.

3. Criteria established by using the direction of the propagation of the pixel containing the maximum ROS value.

The following sections describe in detail the different approaches. We end by discussing the advantages and drawbacks of every different solution coming from them.

\section{Options Based on the Maximum ROS Value}

This option is based on the fact that the head fire perimeter involves the zone of the fire perimeter where the maximum values of the ROS are achieved. According to this, for every isochrone the head fire perimeter has been defined as made by those pixels with a ROS value greater than a given percentage of the maximum ROS value computed at the isochron. As there is not any special reason to select one or another percentage, three values have been tested: $50 \%$ (Fig. 3a), $40 \%$ (Fig. 3b) and $30 \%$ (Fig. 3c). 


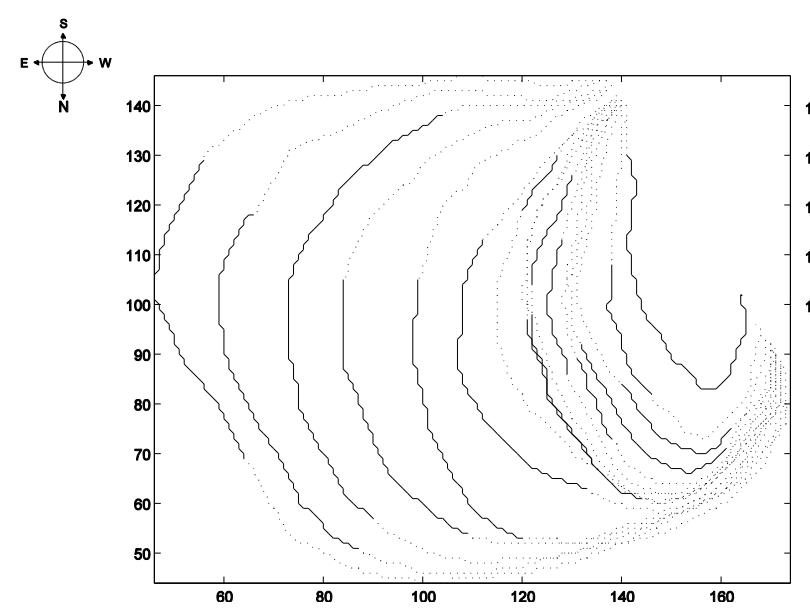

(a)

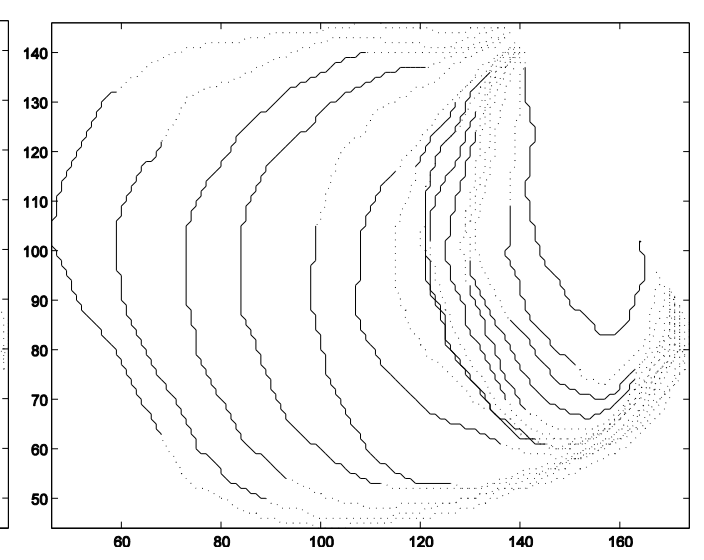

(b)

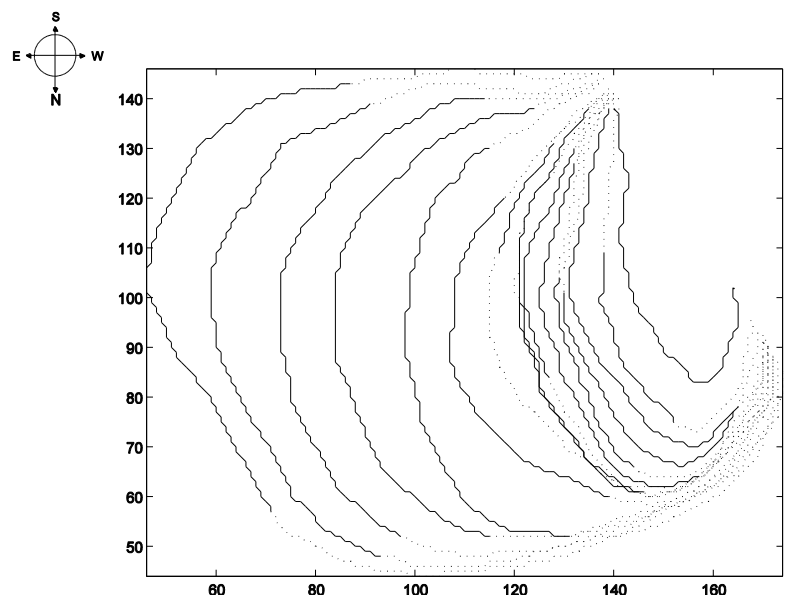

(c)

Fig. 3. Position of the head fire perimeter (black line) with respect to the whole fire perimeter (dotted line) based on the criterion of the maximum ROS value. Isochrones every $30 \mathrm{~s}$ : (a) $50 \%$ criteria; (b) $40 \%$ criteria; (c) $30 \%$ criteria. Values in the axes are in pixels.

Options (a) and (b) in Fig. 3 are quite similar with only slight differences in some of the isochrones. However, option (c) has clearly larger head fire perimeters observed in the entire isochrones, taking apparently part of the flanks. It is really difficult to decide which option suits best the definitions already existing in the literature, as we cannot compare our definitions of the head fire perimeter with what has been proposed by other authors.

We can say that option (c) in Fig. 3 is apparently the one which, at first glance, best represents Cheney and Gould's second definition. However, by using the $30 \%$ ROS criteria, we cannot say that we have systematically identified the point of divergence. Actually, the fact that one isochrone starts to diverge at a given point means that the ROS starts to increase significantly at this point to reach at some stage the maximum ROS value. So if we look at the curve corresponding to the evolution of the ROS along the fire perimeter, the divergence point has to correspond to a change in the slope of this curve.

In Fig. 4 we have plotted these curves for two different isochrones (22 left and 21 right), and we have shown the head fire perimeter delimited by the points $x 1$ and $x 2$, using the three different percentages explained above. For isochrone 22 (Fig. 4, left) the three cases show similar results and in all of them the starting and ending points of the head fire perimeter correspond to a significant change in the slope of the curve. Nevertheless, for isochrone 21 (Fig. 4, right), the $50 \%$ and $40 \%$ cases are relatively similar and in both the starting and ending points of the head fire perimeter correspond to a significant change in the slope of the curve, whereas in the $30 \%$ case there is a clear overestimation of the head fire perimeter. 

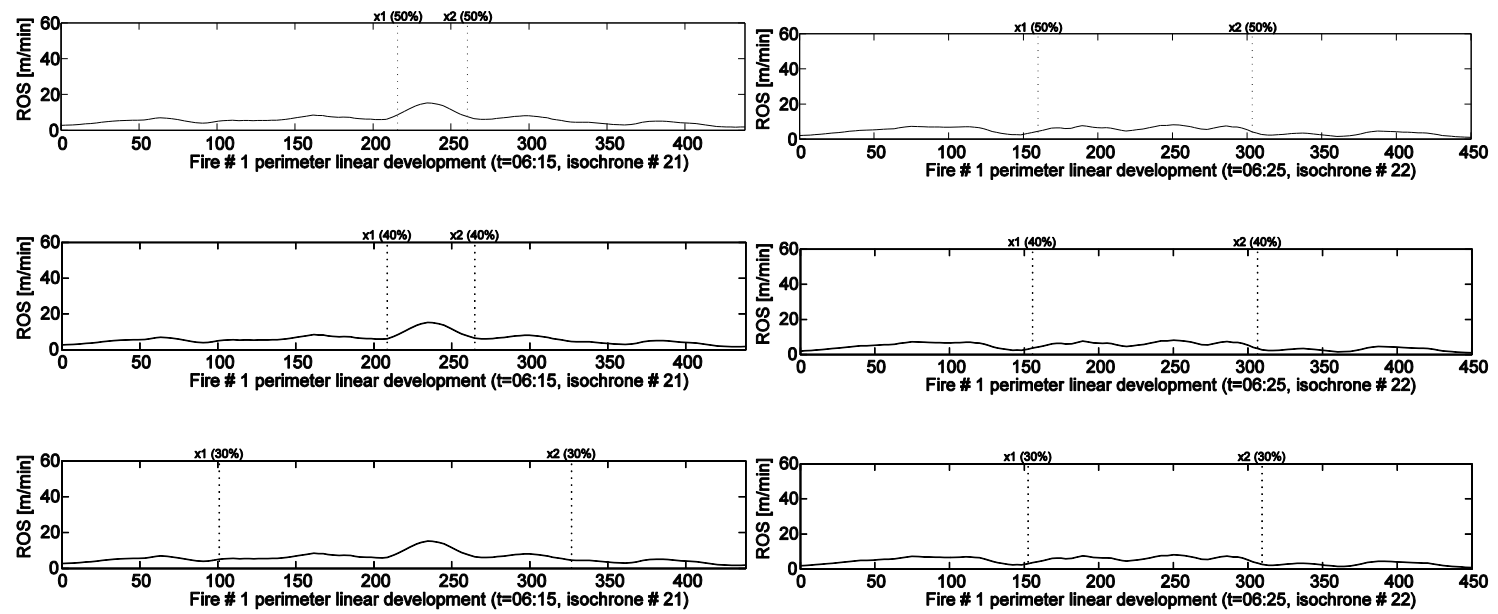

Fig. 4. Evolution of the ROS along the fire perimeter (distance given in meters) for isochrones 22 (left) and 21 (right). Position of the fire front for the three criteria used: $50 \%, 40 \%$ and $30 \%$.

When exploring all the isochrones, generally the $50 \%$ and $40 \%$ criteria tend to be more similar although sometimes the results obtained by the $40 \%$ and $30 \%$ criteria are more alike differing significantly with the results obtained by the $50 \%$ criterion (see for instance isochrones 25 (left) and 37 (right) in Fig. 5).
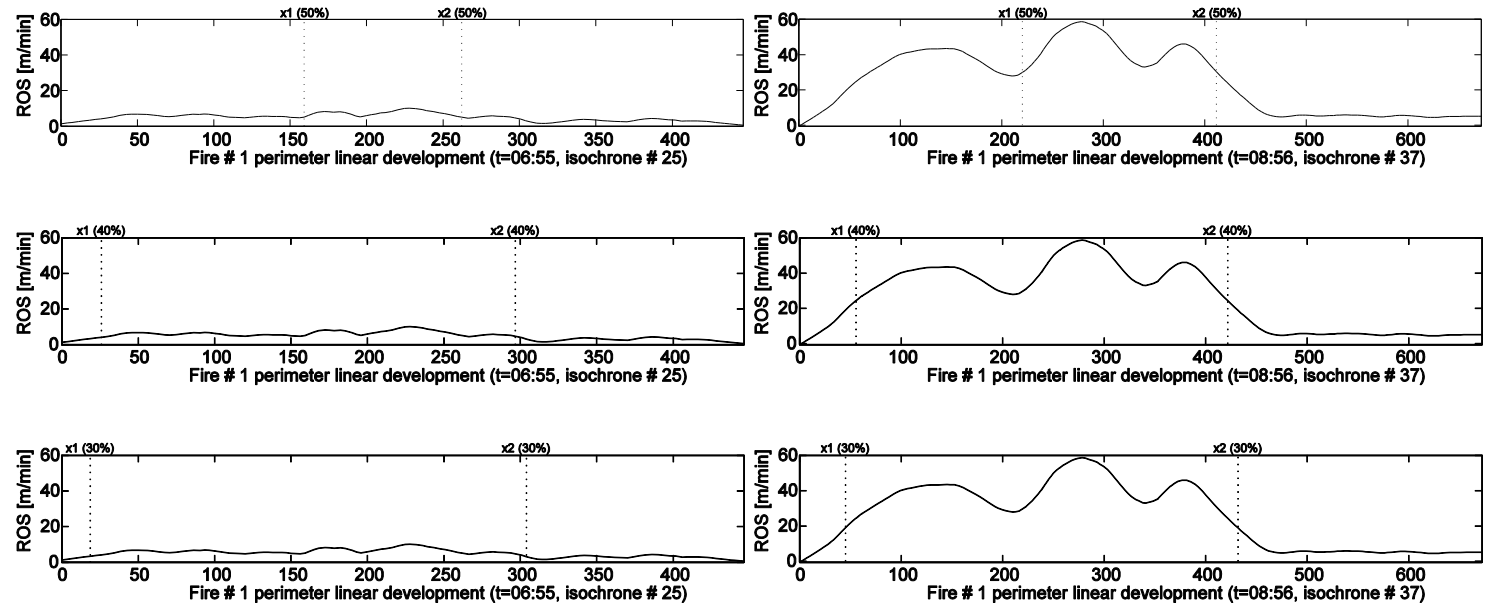

Fig. 5. Evolution of the ROS along the fire perimeter (distance given in meters) for isochrones 25 and 37.

Position of the fire front for the three criteria used: $50 \%, 40 \%$ and $30 \%$.

It is thus difficult to decide which percentage best defines the head fire perimeter. Moreover, there is also a potential problem associated with the fact of fixing a given percentage to delimit the head fire perimeter, since we might be somehow predetermining the values of the head ROS.

Another interesting thing to mention is that when talking about losing the parallelism between isochrones, it will also be helpful to look at the evolution of the distance between one isochrone and its previous one along the fire perimeter. But, as we have plotted the isochrones every $10 \mathrm{~s}$, the graphs would look exactly the same as the ones with the ROS along the fire perimeter just multiplied by 10 , without giving any extrainformation.

\section{Options Based on Derivative of the Isochrones}

With the idea of finding the point with a sudden change in the slope of the curve, the derivative curve of ROS with respect to the fire front development (see Fig. 6) has been analyzed. In this case a certain maximum and a certain minimum of this curve will correspond to our two points of interest $(x 1$ and $x 2)$. 
Nevertheless, here again it is difficult to decide which maximum has to be taken as starting point of the head fire perimeter and which minimum as the finishing point of the head fire perimeter.

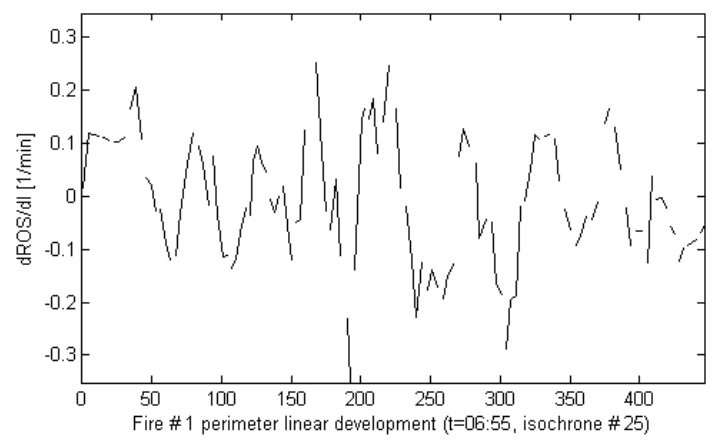

(a)

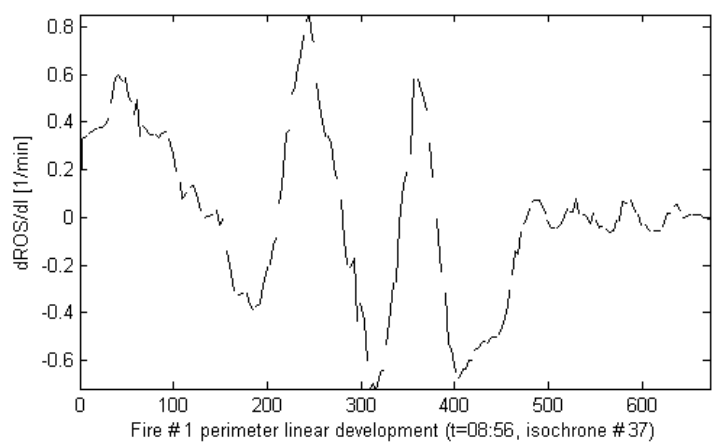

(b)

Fig. 6. Evolution of the ROS derivative along the fire perimeter for isochrones: (a) 25; (b) 37.

According to the Cheney and Gould second definition we could say that the strongest change in the fire ROS should correspond to the maximum value of the derivative, at the starting point of the head fire perimeter, and to the minimum value at the ending point. So, applying this criterion the $x 1$ and $x 2$ values obtained for the isochrones 25 and 37 are shown in Fig. 7.

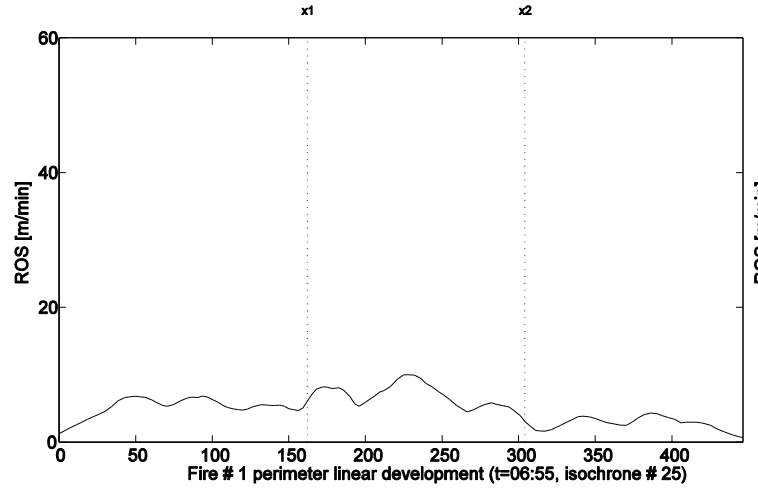

(a)

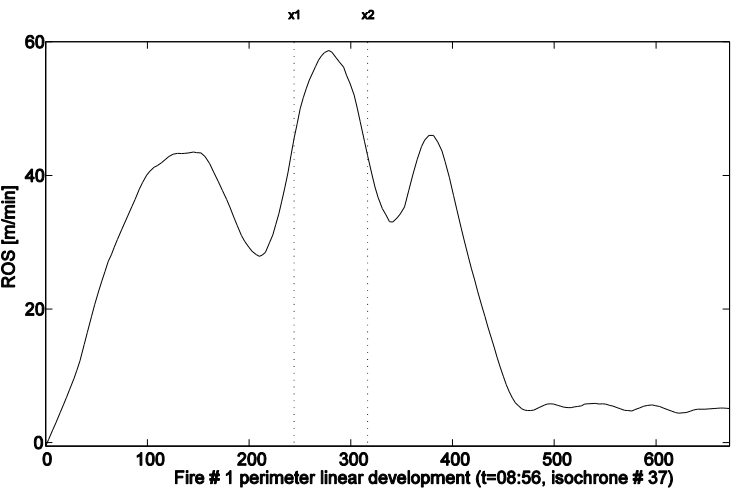

(b)

Fig. 7. Head fire position for the isochrones: (a) 25 and; (b) 37 according to the derivative criterion.

As sometimes a maximum or minimum in the derivative can be found at the beginning or the end of the whole fire perimeter, the first and last $10 \%$ of the fire perimeter - belonging to the flanks - have not been taken into account when searching the absolute maximum and minimum values of the derivative.

In general what can be observed when applying this criterion is that it is often more restrictive than the percentage criterion (see Fig. 8). Nevertheless, the advantage of this criterion is that there is no need of defining an arbitrary threshold value to delimit the head fire perimeter and moreover the subsequent statistical analysis of the head fire behaviour will not be influenced by this threshold. 


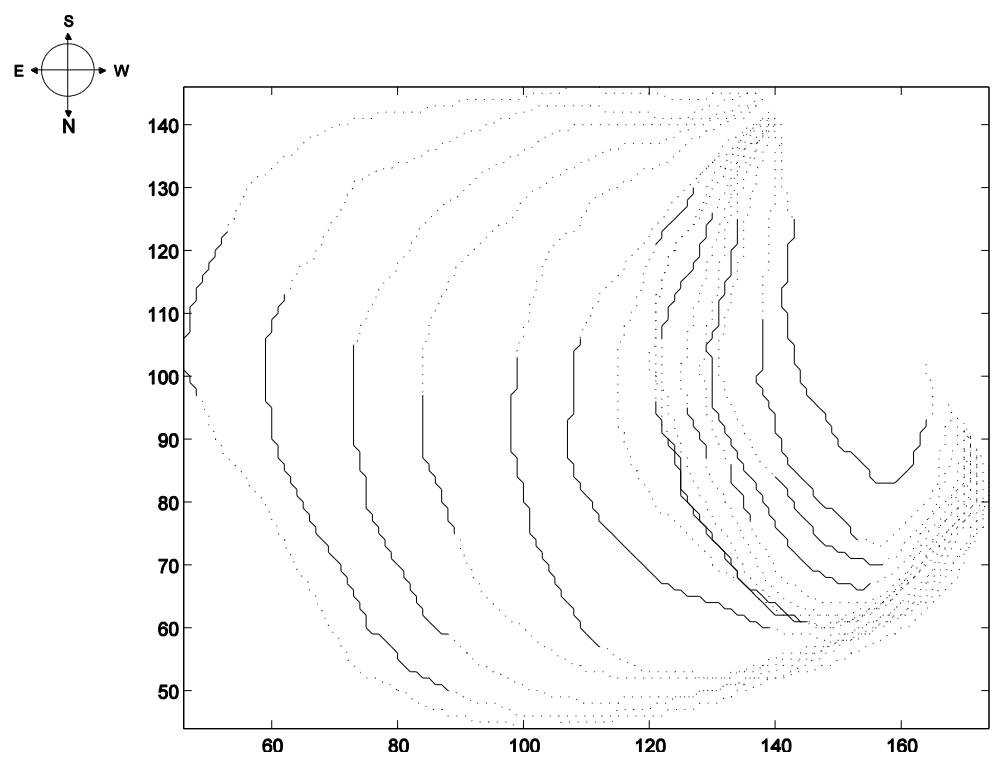

Fig. 8. Position of the head fire perimeter (black line) with respect to the whole fire perimeter (dotted line) based on the criterion of the derivative of the ROS (one isochrone every $30 \mathrm{~s}$ ). Values in the axes in pixels.

\section{Options Based on Direction of the Propagation of the Pixel Containing the Maximum ROS Value}

In this case the head fire perimeter has been defined as all those pixels that have a propagation direction equal to the direction of the pixel that has the maximum ROS, plus or minus a range of degrees. The following values have been tested: $\pm 15^{\circ}$ (Fig 9a), $\pm 30^{\circ}$ (Fig 9b), $\pm 45^{\circ}$ (Fig 9c) and $\pm 60^{\circ}$ (Fig 9d).

Fig. 9 is apparently similar to Fig. 3, maybe with the exception of the figure corresponding to the $\pm 15^{\circ}$ (which gives really very small head fires). However, exploring different isochrones, sometimes there are some inconsistencies when looking in detail at the position of the head fire given by this criterion and comparing to the criterion of the maximum ROS.

As an example, the cases of isochrones 14 and 15, which are consecutive and at only $10 \mathrm{~s}$ of distance are analyzed. In Fig. 10a we have plotted isochrone 14 with the head fire limits $(x 1)$ and $(x 2)$ defined by the $\%$ ROS criteria, whereas Fig. 10b contains the same information using the direction of propagation criteria. In Fig. 10c isochrone 15 is plotted with the head fire limits $(x 1)$ and $(x 2)$ defined by the \%ROS criteria, whereas Fig. 10d has the same information using the direction of propagation criteria. 


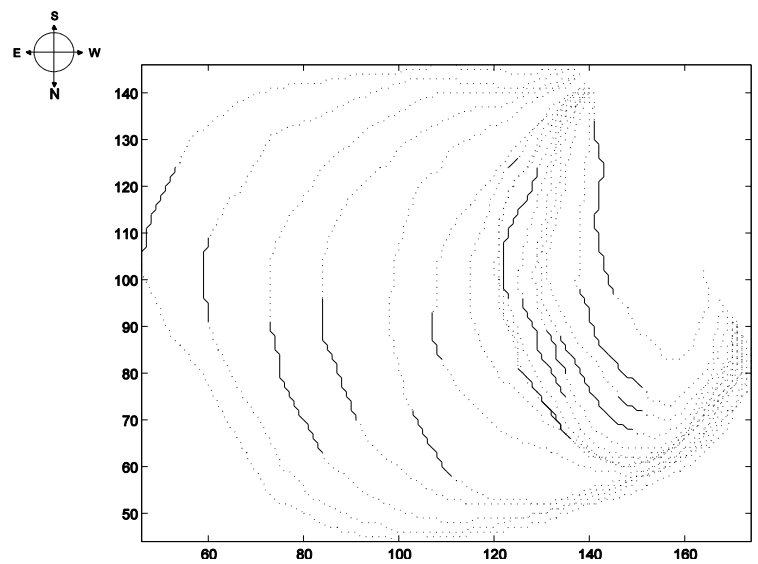

(a)

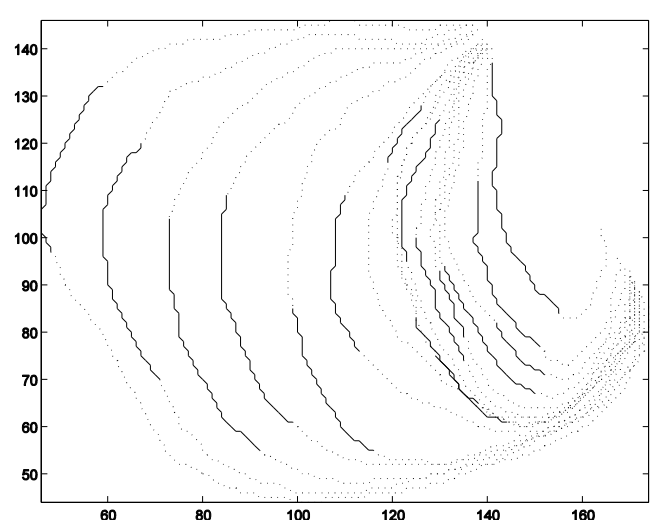

(b)

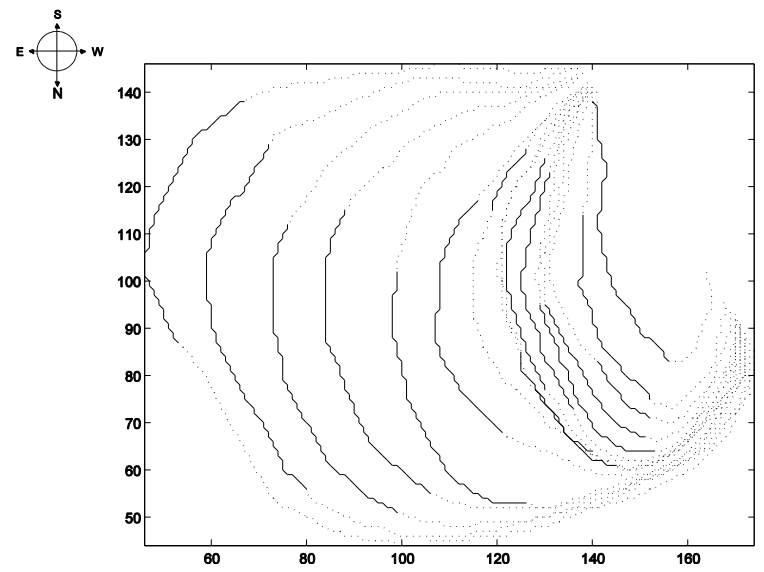

(c)

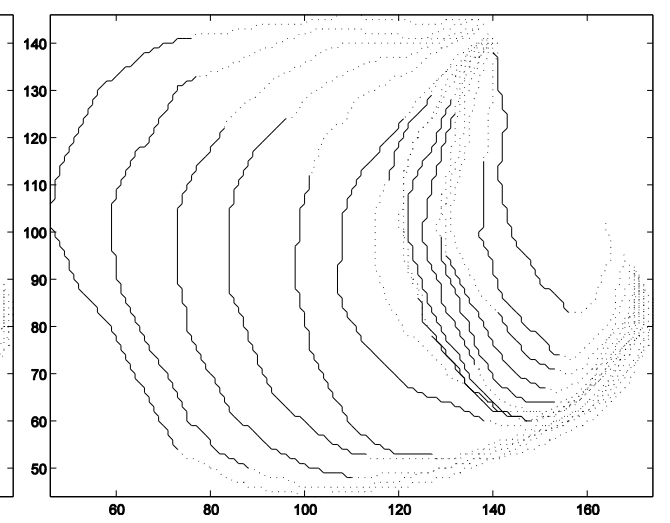

(d)

Fig. 9. Position of the head fire perimeter (black line) with respect to the whole fire perimeter (dotted line) based on the criterion of the propagation direction of the maximum ROS pixel (one isochrone every $30 \mathrm{~s}$ ): (a) $\pm 15^{\circ}$ criteria; (b) $\pm 30^{\circ}$ criteria; (c) $\pm 45^{\circ}$ criteria; (d) $\pm 60^{\circ}$ criteria (numbers in the axes are pixels).

In both isochrones 14 and 15, the \%ROS criteria give the position of the head fire perimeter around the interval $(60 \mathrm{~m}-300 \mathrm{~m})$. However, when observing the results given by the direction criteria we found narrower head fire perimeters located around $(100 \mathrm{~m}-175 \mathrm{~m})$ for isochrone 14 and around $(200 \mathrm{~m}-275$ $\mathrm{m})$ for isochrone 15 . Note here that the position of the head fire perimeter has dramatically changed in only $10 \mathrm{~s}$, which seems to be unrealistic. 

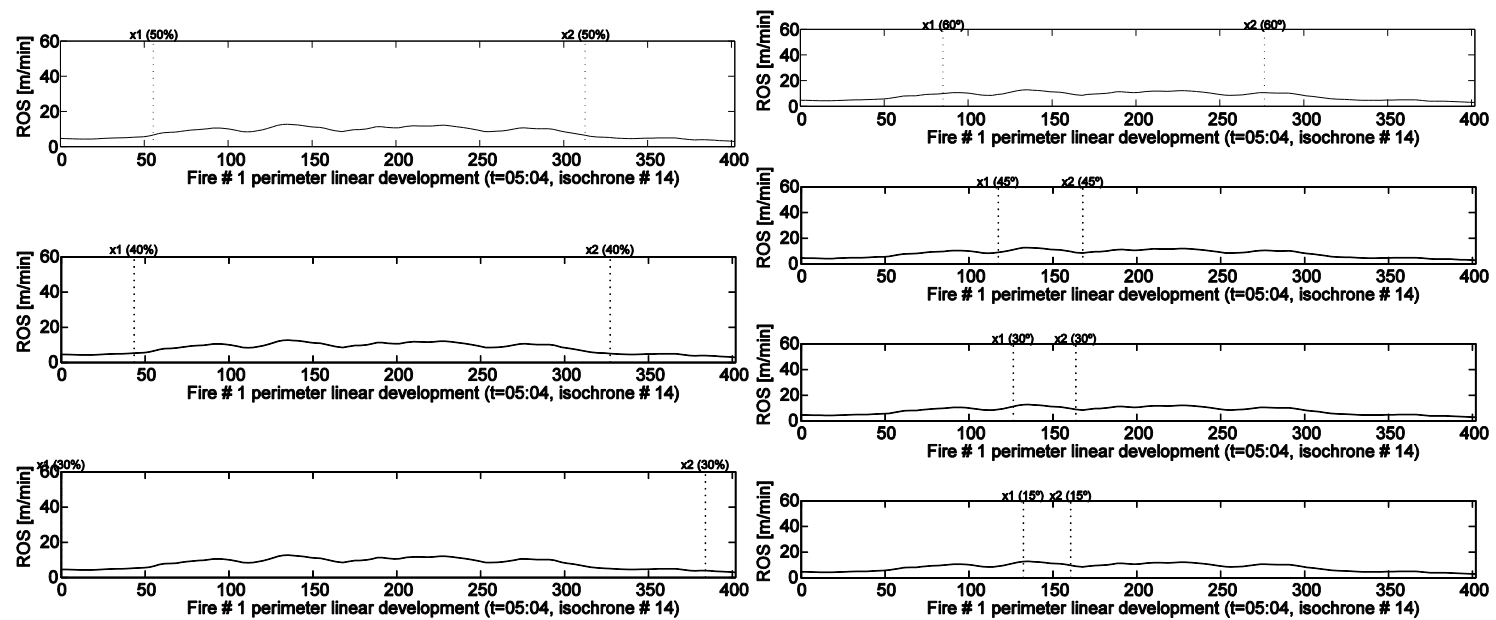

(a)

(b)
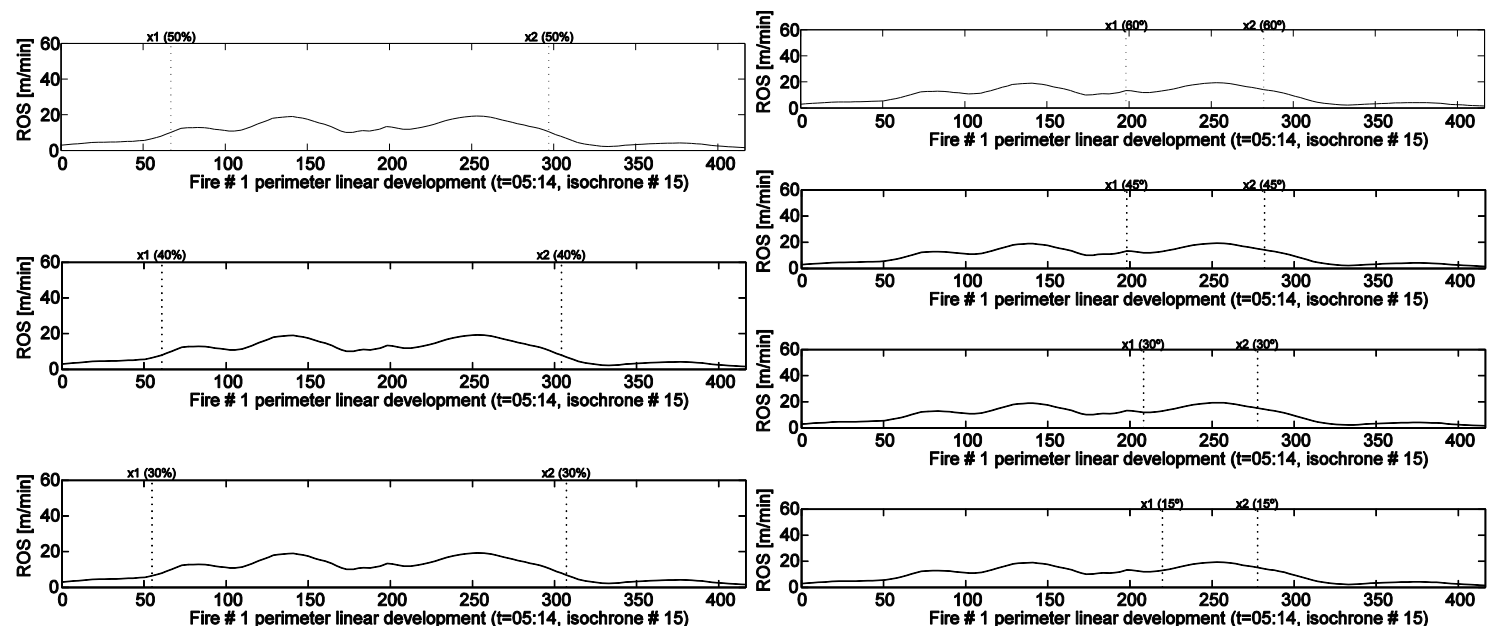

(c)

(d)

Fig. 10. Evolution of the ROS along the fire perimeter (distance in meters). (a) Isochrone 14 and percentages criteria; (b) Isochrone 14 and angles criteria; (c) Isochrone 15 and percentages criteria; (d) Isochrone 15 and angles criteria.

It also has to be highlighted that the propagation direction criterion does not reflect necessarily the initial and final position of the head fire perimeter coinciding with a sudden change in the slope of the curve shown in the linear development of the isochrones.

\section{CONCLUDING REMARKS}

In order to compare the fire front perimeter dimensions according to the different criteria analyzed, Fig. 11 shows the head fire perimeter length for all the isochrones obtained by all the options explored. Generally speaking, all the criteria follow more or less the same trend although the $30^{\circ}$ and $15^{\circ}$ criteria differ more, especially at the last isochrones, showing practically no increase in the head fire length. This might be unrealistic, since an increase in the head fire perimeter should be generally expected as the fire grows. Moreover, for an easy understanding of Fig. 9 a statistical analysis has been performed to every criterion and considering all the isochrones (Table 1). As it can be seen, the $30 \%$ criterion is the one giving the larger head fire lengths while the $15^{\circ}$ and the $30^{\circ}$ criteria are the ones giving usually the smallest lengths. 
Table 1. Summary of the basic statistics performed for all the isochrones and criteria used.

\begin{tabular}{|l|c|c|c|c|c|}
\hline Criteria & Mean & SE mean & Std. dev. & Minimum & Maximum \\
\hline Total length & 527,50 & 27,80 & 188,20 & 266,30 & 957,20 \\
\hline $60^{\circ}$ & 201,00 & 13,90 & 94,10 & 56,20 & 373,90 \\
\hline $45^{\circ}$ & 152,50 & 10,00 & 67,80 & 47,70 & 278,70 \\
\hline $30^{\circ}$ & 109,11 & 6,50 & 44,09 & 37,24 & 185,82 \\
\hline $15^{\mathbf{o}}$ & 64,41 & 4,02 & 27,26 & 8,49 & 120,94 \\
\hline $50 \%$ & 146,60 & 11,30 & 76,80 & 36,00 & 314,90 \\
\hline $40 \%$ & 192,10 & 13,40 & 90,60 & 48,20 & 366,20 \\
\hline $30 \%$ & 250,90 & 14,70 & 99,40 & 51,20 & 419,00 \\
\hline Derivative & 127,70 & 10,70 & 72,50 & 21,00 & 248,50 \\
\hline
\end{tabular}

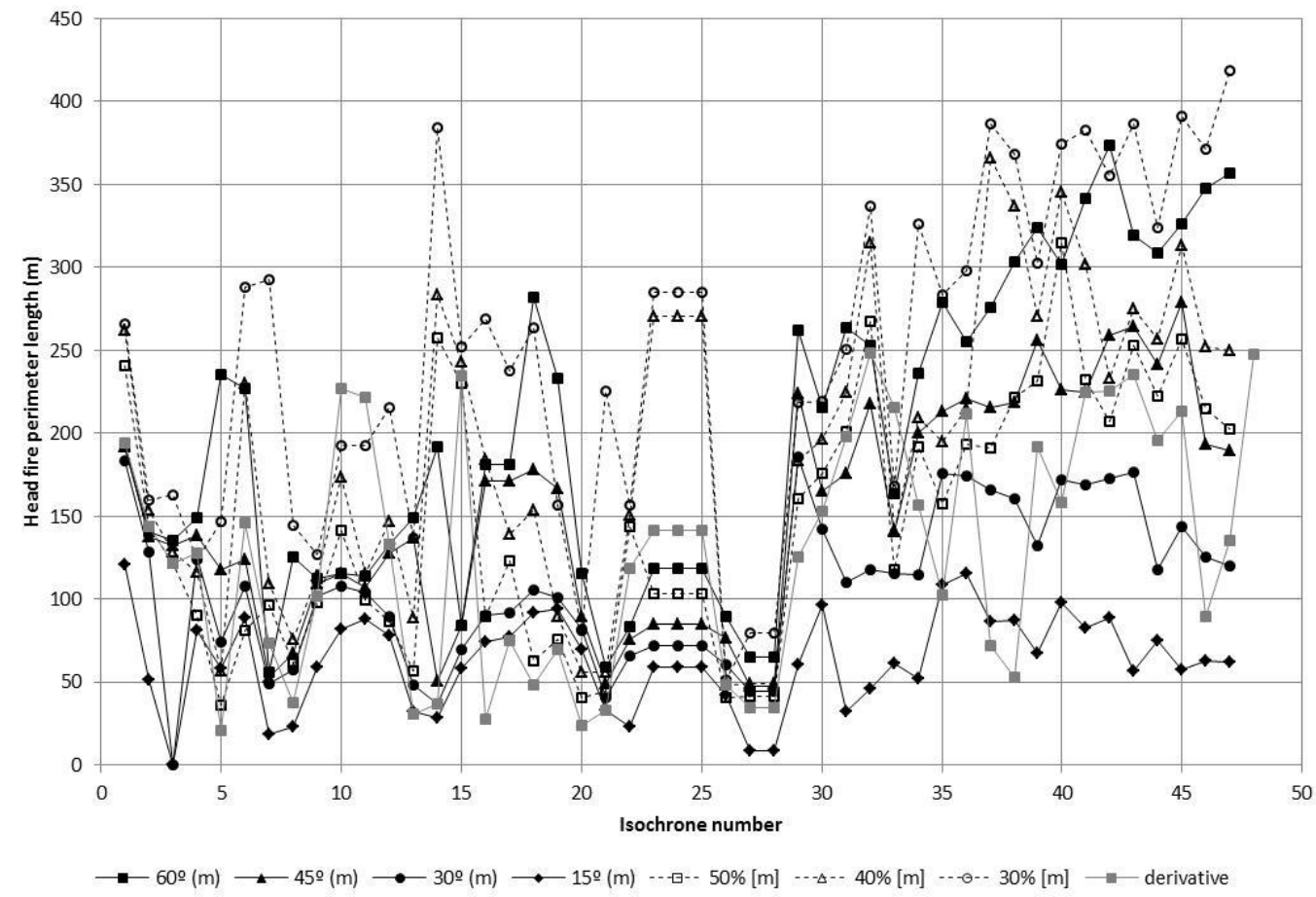

Fig. 11. Comparison of the head fire perimeter length for all the isochrones and all the criteria analyzed.

With the analysis performed, we can say that there is not an ideal solution for the definition of the head fire perimeter since any option requires a more or less arbitrary selection of a threshold to mark the limits between the head and the flanks. The derivative of the isochrones seems to be the option that could better reflect the physical phenomena which take place in the transition between the flanks and the fire head. Nevertheless, it seems to be a lot more restrictive than any other explored option and also than the solution given in [4]. Actually, the option which seems to better represent Cheney and Gould's concept is the criteria based on the percentage ROS.

The following points summarize the options we have come out with, with their advantages and drawbacks:

a) To fix a restrictive value like $50 \%$ of ROS in order to pick the extreme behaviour of the head fire knowing that in some cases the head fire perimeter may be underestimated and that, when computing mean ROS values, the results will be somehow predetermined as all ROS values will be between the maximum ROS and its $50 \%$.

b) To fix a conservative value of the percentage like the $30 \%$ of maximum ROS assuring that all the fire head is taken. In this case some parts of the flanks might be wrongly taken.

c) Taking $x 1$ and $x 2$ as the value obtained averaging the $x 1$ and $x 2$ values corresponding to the three percentages tested. This solution is a bit more complex but avoids the problem of fixing a given 
percentage and the values obtained will always be nearer to the values of the percentages that are more similar between them.

d) Make similar options of a), b) and c) but using in this case the direction of propagation criteria.

e) Determine $x 1$ and $x 2$ by the derivative criteria. This would better reflect the physical phenomena of the flank/head fire behaviour transition with less arbitrariness than the other options. However, it would represent even shorter head fire perimeters than what has classically been understood.

Several definitions of the head fire perimeter have been explored in order to find precise, unequivocal and subjectivity-free criteria to delimit the head of a fire from their flanks for scientific purposes. This study has come out with five different criteria that can be used when analysing fire behaviour for scientific purposes. They are all based on studying the differences in rate of spread (module and direction) along the fire perimeter and they all have different degrees of arbitrariness giving more or less restrictive solutions to the problem. The use of one or other option depends on the final aim of the study being undertaken. So, criteria giving shorter head fire perimeters might be more suitable to study extreme fire behaviour since these options are focussed on picking a narrow range of intense fire behaviour. On the other hand, more conservative options will better suit to studies in which average estimations of fire behaviour variables on larger parts of the fire front are needed.

\section{ACKNOWLEDGEMENTS}

This research is supported by the Spanish Ministry of Education and Science under the project AGL200801161. It is also supported by the European Social Fund, the Technical University of Catalonia (UPC), the Department of Universities, Research and the Information Society (DURSI) of the Autonomous Government of Catalonia. This entity also supports this research under the project No. 2009SGR1118. The experiments were supported by the South Australian Department for Environment and Heritage, the South Australian Country Fire Authority, the Victorian Department of Sustainability and Environment and the Bushfire Cooperative Research Centre. We also wish to thank the many firefighters and research staff who assisted with the experiments. Finally we especially acknowledge the thoughtful comments provided by Miguel Cruz.

\section{REFERENCES}

[1] Van Wagner, C.E., (1969). A simple fire-growth model, Forestry Chronicle 45: 103-104.

[2] Anderson, H.E., "Predicting wind-driven wild land fire size and shape", USDA Forest Service, Research Paper INT-305. 1983, 23 p.

[3] Forestry Canada Fire Danger Group, (1992). "Development and structure of the Canadian forest fire behaviour prediction system". Forestry Canada. Science and Sustainable Development Directorate, Inf. Rep. ST-X-3, 63 p.

[4] Cheney, N.P., and Gould, J.S., (1995). Fire growth in grassland fuels, International Journal of Wildland Fire 5(4): 237-247, http://dx.doi.org/10.1071/WF9950237

[5] Cruz, M.G., Matthews, S., Gould, J., Ellis, P., Henderson, M., Knight, I., and Watters, J. (2010). Fire dynamics in mallee-heath; fuel weather and fire behaviour prediction in South Australian semi-arid shrublands, Bushfire Cooperative Research Centre Technical Report A.10.01. East Melbourne, Victoria.

[6] Plucinski, M., Cruz, M., Gould, J., Pastor, E., Pérez, Y., Planas, E. and McCarthy, G. (2010). Project FuSE Aerial Suppression experiments. Bushfire Cooperative Research Centre Technical Report, East Melbourne, Victoria.

[7] Pastor, E., Pérez, Y., Cubells, M., Planas, E., Plucinski, M. and Gould, J.S., (2010). "Quantifiable assessment of aerial suppression tactics in wildland fires using airborne infrared imagery" $V I$ International Conference on Forest Fire Research, D. X. Viegas (Ed.). 